\title{
Modeling the Discovery of Critical Utterances
}

\author{
Calkin A.S. Montero ${ }^{1}$, Yukio Ohsawa ${ }^{2,3}$, and Kenji Araki ${ }^{1}$ \\ ${ }^{1}$ Graduate School of Information Science and Technology, Hokkaidō University, \\ Kita 14-jo Nishi 9-chome, Kita-ku, Sapporo City, 060-0814 Japan \\ \{calkin, araki\}@media.eng.hokudai.ac.jp, \\ http://sig.media.eng.hokudai.ac.jp \\ ${ }^{2}$ Graduate School of Business Science, University of Tsukuba, \\ 3-29-1 Otsuka Bunkyo, Tokyo, Japan \\ 3 Graduate School of Information Science an Technology, The University of Tokyo, \\ Tokyo, Japan \\ osawa@gssm.otsuka.tsukuba.ac.jp \\ http://www.gssm.de/comp/lncs/index.html
}

\begin{abstract}
The ubiquity of chance discovery can be seen in many areas of application, fundamentally when there are events that are difficult to find or predict. In this paper, we present our idea of applying a chance discovery tool, the KeyGraph, for identifying relationship of co-occurrence between the utterances of the interlocutors during a chat section.
\end{abstract}

\section{Introduction}

Despite of being a new field of research and still in a early stage of development, chance discovery is being applied to a wide range of branches of science. Chance discovery, as a field of investigation itself, is devoted to analyze the occurrence of events that might have important impact in their happening area. In this regard, the word "chance" has been defined as the information about an event or situation significant for making decisions, being chance discovery the discovery of a chance, not by chance [1]. This chance has been considered a rare opportunity - or risk - whose significance has been unnoticed, which may lead to an unexpected benefit.

Such chances have been studied in areas of application such as:

- earthquake prediction

- topic diffusion in communities

- genomes triggering diseases

- new products and consumer's behavior

- complex adaptive systems

- leading opinions in on-line communities

- data mining with visual interface

- discourse analysis

and so forth. In this regard, we applied techniques of chance discovery to process human-human chat ${ }^{1}$.

\footnotetext{
${ }^{1}$ Friendly, informal conversation. Cambridge Advanced Learner's Dictionary.
} 
During the analysis of human-human chat, a peculiar behavior of critical selforganization was observed [2]. It could be considered that the positive feedback effect that exists, from the interlocutors during the chat, triggers the self organization. We argue that for modeling a human-like computer chat this critical behavior should be taken in consideration.

Previous research has approached the problem of modeling computer dialogue [3]. In fact, three main approaches have been proposed to modeling dialogue: dialogue grammars approach, plan-based approach, and joint action or collaborative approach. The dialogue grammar approach is based on the observation that sequencing regularities appear in the dialogue [4] - so as questions are generally follow by answers, proposal by acceptances, etc -. Hence, it has been proposed that dialogues are a collection of such act sequences with embedded sequences of digressions and repairs. This kind of grammars can be constructed following the Chomsky hierarchy or finite state machines. The plan-based approach is based on the observation that the user's utterances are not simple string of words but communicative actions or speech acts [5]. This approach assumes that the user's speech acts are part of a plan, and the goal of the system would be to uncover and respond appropriately to this plan [6]. Finally, the joint action or collaborative approach views the dialogue as a collaboration between the interlocutors in order to achieve a mutual understanding [7]. All of those approaches focused on creating a dialogue in order to achieve certain specific goal, it is to say, domain specific dialogue approaches.

When it comes to modeling non-specific-goal computer dialogue ${ }^{2}$, in spite of the increasingly development of such dialogue agents, there is a remarkable lack of research. Non-specific-goal dialogue agents are called chat robots or chatbots. A chatbot has been defined as a computer program that simulates human conversation through Artificial Intelligence (AI). The dialogue modeling of a chatbot is based on certain words combinations that it finds in a phrase given by the user, and in the case ALICE (Artificial LInguistic Computer Entity) [8] chatbot, $\mathrm{AIML}^{3}$ is used to transcribe pattern-response relations. However, the chatbot often gets lost during chatting with a user since it is highly improbable to create all the possible pattern-response. In this research we applied a chance discovery tool, the KeyGraph, in order to observe the critical behavior and dynamics of the human chat, information that, we argue, is of great value for meliorating the modeling human-like computer chat.

\section{Human-Human Chat Dynamics: A Chance}

While researching on the structure of human-human chat, we observe a peculiar general behavior in the flow of the conversation. Let us explain our observations as follow: considering a trivial conversation - chat - between two interlocutors, it is noticeable that in the beginning the chat could be considered flat (greetings from each interlocutors). As the chat evolves toward one direction - some

\footnotetext{
${ }^{2}$ Open domain dialogue.

3 Artificial Intelligence Markup Language, base on extended Markup Language, XML.
} 
specific topic - certain utterances may cause the course of the chat to slightly chance the direction of the topic, although the initial topic still remains the same. Eventually, during the chat there is not anything else to utter about the initial topic, so this subject can not be discussed any further, either because the interlocutors have already agreed in their ideas regarding the subject or because the topic is not longer of interest for the interlocutors. At this point a single utterance given by any of the interlocutors can cause the whole direction of the chat to change toward a new topic and the whole process starts again. Making an analogy with self-organized criticality exemplified by the Bak's sandpile model [9], this behavior has been called critically self-organized chat (CSOC) [2]. The above described behavior can be seen in the following fragment of a real chat between two friends ${ }^{4}[10]$ :

1A: Hello

1B: Hello A, ah

2A: Yes, B ah

2B: Ah

3A: Have you had your lunch

3B: No

4A: Uhm I haven't had my lunch also

4B: Uh busy uh

5A: Uhm busy playing with my nieces

$5 \mathrm{~B}$ : Both of them are in their own place eh at your home now

6A: Hah The younger one is always at my home mah

6B: Both of them - Orh

39A: You hear my little niece crying

39B: Heh uh Uhm so are you

spring cleaning already

40A: Ah intend to do it today lor

40B: Orh
41A: So I will be home whole day lah but 41B: Uhm

42A: Uhm you know like can't get started like that do a little bit then no don't want to do uhm then

42B: Uh huh uh huh

52A: Ah - Then weekdays definitely can not - Weekdays I'm so busy in the office you know

52B: Ah hah

53A: This whole this week itself ah

53B: Ah hah

54A: Every night you know

54B: Ah

55A: Past nine o'clock then I leave

the office

55B: Whoa everyday

In this example, the utterances $1 \mathrm{~A}$ to $4 \mathrm{~B}$ form the flat initial state of the system: greetings and topic-introductory utterances. At this point there are not big changes in the chat, it could be said the system is in equilibrium. The next utterance - 5A - initiates the chat toward a determined topic: A's nieces. This topic remains the main one, although it might get slightly changed, until the utterance 39B that "crashes" this main topic initiating a completely new one: "laundry on weekends". And then again, utterance 52A "crashes" the previous topic and started a new one: "busy at the office". This process repeats itself until the chat ends. The utterance that changes the direction of the chat - crashing one topic and starting another - is called "critical utterance" [2].

Considering the flow of a chat, it can be said that the chat is a dynamic system whose environment is formed by the interlocutor's utterances. In this

\footnotetext{
${ }^{4}$ The speakers names have been omitted.
} 
dynamic system a chance could be seen as the moment when a critical utterance may come as to define the new direction of the chat.

\section{Chance Discovery and Critically Self-organized Chat}

It is precisely the dynamic structural behavior of human-human chat that allows the smooth transition from one topic to the other within a chat section. If we consider the time of transition between topics as potential chances, the analysis of critical utterances - when a topic changes - and their relationships becomes relevant. In this regard the application of chance discovery techniques, as data mining processing tool, are considered useful. However, it has been stated in [11] the difficulty of identifying a chance in complex systems - critically selforganized systems - due to the interrelation between the system and its environment. Therefore, a chance must be considered from the global collective nature of the dynamics of the system.

In order to analyze the dynamics of our system - a chat section - as to model how to identify potential chances - critical utterances or topic changing - a chance discovery graphical tool is needed. In this regard, the application of the KeyGraph becomes suitable. The KeyGraph, originally used for indexing documents [12], has developed as a data mining tool for extracting patterns of the appearance of chance events. As a data mining tool, it identifies relationship between terms in a document, particularly focusing on relationship of co-occurrence of both high probability and low probability events. A detailed description of the application of the KeyGraph for analyzing the chat section shown in Sect. 2 is given hereunder.

\subsection{Design Experiment and Visual Results}

We aim at visualizing potential chances during a human-human chat section in order to apply this knowledge to model computer chat. The used tool, the KeyGraph, has been applied to a variety of topics [13]:

- discovering deep building blocks for genetic algorithms

- discovering emerging topics from the World Wide Web

- discovering areas with high risk of near-future earthquakes

and so forth. In our research, it is used to visualize critical utterances.

The analysis was carried out as follow:

1) Each turn of the speakers - utterance - was considered one sentence.

2) Each sentence was segmented by words.

3) High frequency words were eliminated, i.e., I, you, are, my, and the like.

4) A vectorial representation of each sentence was created in order to find cooccurrence relation between them.

5) The co-occurrence document was analyzed using the KeyGraph

Let us suppose our chat section, $C_{S}$, as a matrix where columns represent words, $w_{n}$ for $n=1,2,3, \ldots, k$, and rows represent sentences, $S_{m}$ for $m=$ $1,2,3, \ldots, l$, in the form: 
$C_{S}=$

\begin{tabular}{ccccc|c}
$w_{1}$ & $w_{2}$ & $w_{3}$ & $\cdots$ & $w_{n}$ \\
0 & 0 & 1 & $\cdots$ & 1 & $S_{1}$ \\
1 & 0 & 1 & $\cdots$ & 1 & $S_{2}$ \\
0 & 1 & 0 & $\cdots$ & 0 & $S_{3}$ \\
& & $\vdots$ & & & $\vdots$ \\
0 & 1 & 1 & $\cdots$ & 0 & $S_{m}$
\end{tabular}

where the zeros and ones represent the absence or presence of a word in each sentence. Transforming this document by obtaining the transposed matrix, it becomes:

$$
\left(C_{S}\right)^{T}=\quad \begin{array}{ccccc|c}
S_{1} & S_{2} & S_{3} & \cdots & S_{m} \\
0 & 1 & 0 & \cdots & 0 & w_{1} \\
0 & 0 & 1 & \cdots & 1 & w_{2} \\
1 & 1 & 0 & \cdots & 1 & w_{3} \\
& & & \vdots & & \\
\vdots \\
1 & 1 & 0 & \cdots & 0 & w_{n}
\end{array}
$$

In $\left(C_{S}\right)^{T}$ each row represents what words each sentence shares with each other, what we regard as the occurrence relationship between sentences. As a result, from the above matrix we obtain:

$D=$

$$
\begin{aligned}
& w_{1}: S_{2} \\
& w_{2}: S_{3}, S_{m} \\
& w_{3}: S_{1}, S_{2}, S_{m} \\
& \cdots \\
& w_{n}: S_{1}, S_{2}
\end{aligned}
$$

This document $D$ is then analyzed using the KeyGraph.

Applying the above described algorithm to the chat section the graphical result is given in Fig. 1.

In this figure the results of the KeyGraph can be interpreted as follow:

- Each one of the dotes represent one sentence.

- Each cluster, formed by groups of dotes, represent the relationship between the sentences that form one specific topic.

- Each link, represent interconnection between topics.

In the analyzed chat there were eleven critical utterances as shown in Table 1. From those, ten (except 78) were identified in the graph - marked with arrows. Observing the clusters in the figure, it can be said that one critical utterance is leading to the other. This might be happening because of the interlocutor's desire (unconsciously) to organize his/her own mind (or schedule for doing things). This desire comes from a natural tendency of human self-discovery and coherence in their own chats. The time when a critical utterance arrives (when the topic under scope loses its novelty, so there is not more interest on it from the 


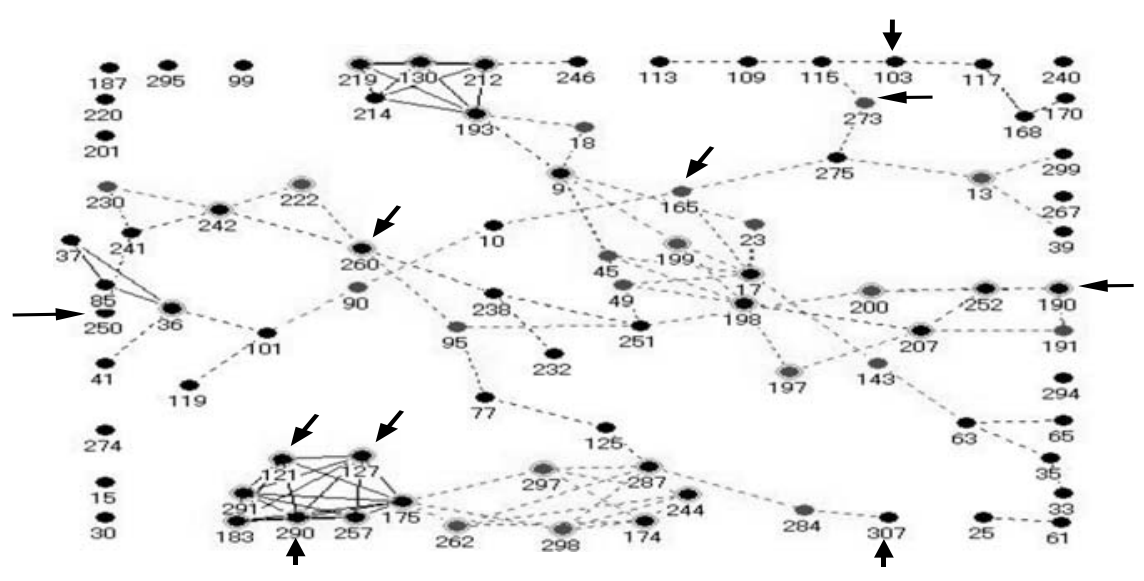

Fig. 1. Graphical View: Human-Human Chat.

participants) is triggering the different links and nodes given by the tool for the critical utterances. However, a topic may have lost its novelty temporarily, but later on the same topic may arise again from another point of view - what makes very difficult to distinguish what is a critical utterance and what is not-, giving birth to the clusters that contain more than one critical utterance all together with other utterances.

The links the graph are representing different ways of transition between one topic and the other. For example, the cluster containing the critical utterances 127 and 290 (concerning about New Year) is linked to utterance 287 (about bringing one of the speaker's niece out) through 297 (about "bring something"). Therefore, the bridges might be indicating transition between one topic and the other, information that can be used for smartly changing topics in a very smooth way when modeling computer chat, i.e., a topic regarding New Year can be shifted, although related, by asking or giving a question like "what are you bringing for New Year's party?".

Although the KeyGraph did not identified all of the critical utterances, the results shown by the graph reflect the dynamic behavior that characterize the tendency to critically self-organize of the human-human chat.

\section{Conclusion}

In this paper an algorithm for modeling the discovery of critical utterances and their relationship within a chat section, was described. Making used of the visual computer aid the KeyGraph the dynamics of the chat were appreciated graphically. In the graph, most of the utterances that caused a change in the topic during the chat section were observed. Those utterances were forming clusters of topics and the links between clusters were representing different ways of transition from one topic to the other. Future works are oriented toward the application of the described algorithm for analyzing and improving computer chat. 
Table 1. Critical Utterances.

\begin{tabular}{|l|l|l|}
\hline Old Topic & Critical Utterance & New Topic \\
\hline Speaker's nieces & 78:So are you spring cleaning already & laundry weekends \\
\hline laundry weekends & 103:weekdays.. I'm so busy in the office .. & busy in office \\
\hline busy in office & 121:the end of next week ..enjoy the New Year & relaxing at home \\
\hline relaxing at home & 127:Chinese New Year, who are you going visit & Chinese New Year \\
\hline Chinese New Year & 165:So you mean we have lunch at your place & meeting New Year \\
\hline meeting New Year & 190:Did I tell you I bought a game set & game \\
\hline game & 250:but the girls will dominate as usual & mix meeting \\
\hline mix meeting & 260:When you see C.. you know what to do right & bringing presents \\
\hline bringing presents & 273:lunch time is it & having lunch \\
\hline having lunch & 290: Okay, have you done New Year shopping & shopping \\
\hline shopping & 307: Okay, so talk again tonight & end of chat \\
\hline
\end{tabular}

\section{References}

1. Ohsawa Y: Modeling the Process of Chance Discovery. In: Ohsawa Y. and McBurney P (eds) Chance Discovery. Springer, Berlin Heidelberg New York. (2003)

2. Montero C.A.S., Araki K.: Discovering Critically Self-Organized Chat. The Fourth IEEE International Workshop on Soft Computing as Transdisciplinary Science and Technology. To appear. (2005)

3. Cohen Phil: Dialogue Modeling. In: Cole R, Mariani J, Uszkoreit H, Varile G, Zaenen A, Zampolli A, and Zue V (eds) A Survey of the State of the Art in Human Language Technology. Cambridge University Press, Cambridge. (1998)

4. Sacks H, Schegloff E, Jefferson G: A Simplest Systematics for the Organization of Turn-taking in Conversation. In: Schenkein J, (editor) Studies in the Organization of Conversational Interaction. Academic Press, New York. (1978)

5. Searle RJ: Speech Acts: An essay in the Philosophy of Language. Cambridge University Press, Cambridge. (1969)

6. Allen FJ, Perrault RC: Analyzing Intention in Dialogues. Artificial Intelligence, 15(3):143-178. (1980)

7. Clark HH, Wilkes-Gibbs D: Referring as a Collaborative Process. Cognition, 22:139. (1986)

8. Wallace RS: A.L.I.C.E. Artificial Intelligence Foundation. http://www.alicebot.org

9. Bak P: How Nature Works, Oxford University Press. (1997)

10. University of South California, Dialogue Diversity Corpus, Dialogue 91,

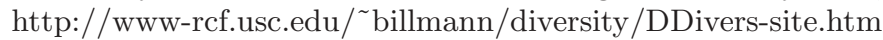

11. Jensen HJ: Self-organizing Complex Systems. In: Ohsawa Y, McBurney P (eds.) Chance Discovery. Springer, Berlin Heidelberg New York. (2003)

12. Ohsawa Y, Benson NE, Yachida M: KeyGraph: Automatic Indexing by Cooccurrence Graph Based on Building Construction Metaphor. Proc. of Advanced Digital Library Conference, pp. 12-18. (1998)

13. Ohsawa Y, McBurney P: Chance Discovery. Springer, Berlin Heidelberg New York. (2003) 